\title{
On Using Random Walks to Solve the Space-Fractional Advection-Dispersion Equations
}

\author{
Zhang Yong, ${ }^{1,2}$ David A. Benson, ${ }^{2}$ Mark M. Meerschaert, ${ }^{3}$ and Hans-Peter Scheffler ${ }^{4}$
}

Received August 16, 2005; accepted February 15, 2006

Published Online: April 5, 2006

\begin{abstract}
The solution of space-fractional advection-dispersion equations (fADE) by random walks depends on the analogy between the fADE and the forward equation for the associated Markov process. The forward equation, which provides a Lagrangian description of particles moving under specific Markov processes, is derived here by the adjoint method. The fADE, however, provides an Eulerian description of solute fluxes. There are two forms of the fADE, based on fractional-flux (FF-ADE) and fractional divergence (FD-ADE). The FF-ADE is derived by taking the integer-order mass conservation of non-local diffusive flux, while the FD-ADE is derived by taking the fractional-order mass conservation of local diffusive flux. The analogy between the fADE and the forward equation depends on which form of the fADE is used and on the spatial variability of the dispersion coefficient $D$ in the fADE. If $D$ does not vary in space, then the fADEs can be solved by tracking particles following a Markov process with a simple drift and an $\alpha$-stable Lévy noise with index $\alpha$ that corresponds to the fractional order of the fADE. If $D$ varies smoothly in space and the solute concentration at the upstream boundary remains zero, the FD-ADE can be solved by simulating a Markov process with a simple drift, an $\alpha$-stable Lévy noise and an additional term with the dispersion gradient and an additional Lévy noise of order $\alpha-1$. However, a non-Markov process might be needed to solve the FF-ADE with a space-dependent $D$, except for specific $D$ such as a linear function of space.
\end{abstract}

KEY WORDS: Random walk, forward equation, fractional advection-dispersion equation, adjoint method

PACS: $02.50 . \mathrm{Ga}, 02.60 . \mathrm{Nm}, 02.60 . \mathrm{Cb}, 05.40 . \mathrm{Fb}$

\footnotetext{
${ }^{1}$ Division of Hydrologic Sciences, Desert Research Institute, Reno, NV 89512, USA; e-mail: yong.zhang@dri.edu.

${ }^{2}$ Department of Geology and Geological Engineering, Colorado School of Mines, Golden, CO 80401, USA.

${ }^{3}$ Department of Mathematics and Statistics, University of Otago, Dunedin, New Zealand.

${ }^{4}$ Department of Mathematics, University of Nevada, Reno, NV 89557, USA.
} 


\section{INTRODUCTION}

The space-fractional advection-dispersion equation (fADE), in which the dispersive flux is described by a fractional space derivative, has been applied to modeling the anomalous or super diffusion of solutes observed in heterogeneous porous media (e.g., Refs. 2, 3, 4, 16, 24, 25). An excellent review of theoretical modeling and applications of superdiffusive processes from the nanoscale in biological systems to very large-scale geophysical, environmental, and financial systems was given recently by Metzler and Klafter. ${ }^{(27)}$ The upswing of the applications of fractional dynamics during the past few years mandates the exact or closely approximate solution of the fractional partial differential equations (PDEs), especially for the numerical solutions required for more realistic cases. This study focuses on the numerical solutions of the fADE in one-dimension and is motivated by observations of anomalous dispersion in porous media. The particle tracking method we use relies on identifying the forward equation for the associated Markov process, and simulating that process from its Langevin equation. Since these forward equations are space-fractional Fokker-Planck equations, the results here should be useful in the broader context of those equations. ${ }^{(27)}$ Note that timefractional Fokker-Planck equations have also been considered, ${ }^{(27)}$ but those equations are not related to any Markov process, and their particle tracking solutions require very different methods. ${ }^{(28)}$ Currently, several numerical methods extended from traditional numerical methods have been proposed to solve the fADE when the transport coefficients vary in one or more dimensions, including the method of lines, ${ }^{(19)}$ the finite element method, ${ }^{(32)}$ and the implicit Euler finite difference method. ${ }^{(21)}$

The random walk particle tracking approach, in which the individual particle movements are certain Markov processes, has been demonstrated by numerous authors during the last two decades to be more computational efficient than traditional numerical methods, such as finite element, finite difference, and characteristic methods, when solving the traditional second-order advection-dispersion equation (ADE). The particle tracking approach does not need spatial discretization ${ }^{(13,17)}$ and it does not suffer the numerical dispersion problem. ${ }^{(15)}$ The superiority of the random walk method to the standard numerical techniques is especially apparent when we simulate solute transport using regional-scale and high-resolution numerical models (e.g., Ref. 39). In addition, the random walk approach does not modify (such as truncate) the target partial differential equations, and thus it does not modify the nature of the physical problem. Furthermore, the stability and convergence analysis required by the traditional numerical methods are not needed. Most importantly, the random walk particle tracking approach can not only solve the PDE, but also improve our understanding about the physical process by providing a description of the dynamics underlying the target PDE. However, this method has not yet been extended to the fADE. 
This study examines the random walk particle tracking approach to solve the one-dimensional fADEs. First, we derive and compare the forward equations for the associated Markov process and the possible forms of fADEs. Then we build appropriate Markov processes to control the particle motions and use the particle number density to solve the fADEs. Especially, we focus on the fADEs with space-dependent velocity and dispersion coefficients since they are more realistic and there are no analytical solutions. Numerical examples are also presented as demonstrations.

\section{THE FORWARD EQUATION AND THE FADE}

To force the random-walking particles to move in the same way that a parcel of solute moves in porous media, we have to control the dynamics of particles based on mean groundwater velocity and the velocity deviations embodied in the fractional-order dispersion term. It is the only way to make the particle number density and the solute concentration satisfy a same form of partial differential equation.

There are several steps to tracking random walkers (or particles) to solve the fADEs in this study. First, we explore the relationship between the dynamics of random-walking particles and the resultant particle number density. Second, given a certain forward equation, we can generate the corresponding Markov process; and vice-versa. Third, we explore the possible forms of fADEs when the mean flow velocity and dispersion coefficient are space dependent. Different forms of the fADE arise that describe different transport behaviors of solutes, and thus may require different Markov processes for particle tracking. The last step is the analogy analysis of the forward equations and the fADEs, or the prescription of the dynamics of particles based on the known or pre-specified groundwater velocity and dispersion coefficients. These steps are discussed in this section.

\subsection{The Markov Process and the Associated Forward Equation}

The non-linear Langevin equation containing a Brownian motion (i.e., Ref. 31, p. 44) can be extended to a general case

$$
d X(t)=A(x) d t+B(x) d L_{\alpha}(t),
$$

where $d X(t)[L]$ is a differential distance of travel, $A\left[L T^{-1}\right]$ is a drift term, $X=X(t)$ is the current state (particle location), $d t[T]$ is a differential unit of time, $B\left[L T^{-1 / \alpha}\right]$ is a term defining the strength of diffusion, and $d L_{\alpha}(t)\left[T^{1 / a}\right]$ is a standard Lévy $\alpha$-stable noise, the increment process of a standard $\alpha$-stable Lévy motion $L_{\alpha}(t)$. Note that the parameters $A$ and $B$ related to the particle dynamics are not necessary the same as the water mean velocity and dispersion coefficient parameters belonging to solute transport in porous media. Also note 
here we use the It $\hat{o}$ (not the Stratonovich) definition of the stochastic integral, to be consistent with standard random-walk methods. The difference between the It $\hat{o}$ and the Stratonovich stochastic integrals and the choice of the Itô for standard random-walk methods is discussed in Ref. 18. The different definitions of the stochastic integral relate to different physical processes.

By extending the classical integer-order adjoint method (Ref. 8, Volume II, p. 338) to fractional orders, we find that the non-linear Langevin process (1) corresponds to the following forward equation (see (A.5) in Appendix A1):

$$
\frac{\partial P}{\partial t}=-\frac{\partial}{\partial x}(A P)+\bar{D} \frac{1+\beta}{2} \frac{\partial^{\alpha}}{\partial x^{\alpha}}\left(B^{\alpha} P\right)+\bar{D} \frac{1-\beta}{2} \frac{\partial^{\alpha}}{\partial(-x)^{\alpha}}\left(B^{\alpha} P\right),
$$

where $\alpha$ is the order of stability of the standard Lévy noise $d L_{\alpha}(t), \beta$ is the skewness, $P$ is the transition probability density which is also the number density of particles, ${ }^{(17)} B^{\alpha}=\operatorname{sign}(B) \cdot|B|^{\alpha}$ where the $\operatorname{sign}(B)$ function is 1 for $B>0$ and -1 otherwise, and $\bar{D}=-1 / \cos (\pi \alpha / 2)$. In this study, we consider the case $1<\alpha<2$. A similar forward equation was also derived by Yanovsky et al. ${ }^{(40)}$ using the Langevin approach. To simulate the anomalously rapid transport of contaminants in porous media, we anticipate that the largest particle motions are in front of the mean, so that $\beta=1$, and thus the forward equation is of the form

$$
\frac{\partial P}{\partial t}=-\frac{\partial}{\partial x}(A P)+\bar{D} \frac{\partial^{\alpha}}{\partial x^{\alpha}}\left(B^{\alpha} P\right) .
$$

Note that the sign of the fractional dispersion term in (3) is negative if $0<\alpha<1$. Equation (2) reduces to the classical second-order Fokker-Planck equation when $\alpha=2$.

The choice of $\beta=1$ is also discussed and used by Refs. 2, 3, 4, 35. As demonstrated by field tests (e.g., Ref. 16), the contaminant transport through fractal porous media tends to exhibit the maximum skewness, possibly due to the fast transport of solute through preferential flow paths at all scales. The singleside fractional derivative in (3) is different from the Riesz space-fractional derivative $\partial^{\alpha} / \partial|x|^{\alpha}$, which is symmetric (e.g., Refs. 12). This symmetric fractional derivative is a special case of (2) with $\beta=0$. The random walks developed here can be extended to the case with two-side fractional derivatives, where the Lévy random noise may have weighted probabilities on both sides (not limited to be symmetric).

We remark that it is also possible to develop variable coefficient transport equations using a continuous time random walk (CTRW) model, see Refs. 7, 28).

\subsection{The Derivation of the fADE}

The advection-dispersion equation describes the transport of dissolved plumes, and can be derived following the mass conservation of solutes within 
a representative element volume fixed in space. The conservation of mass caused by diffusive flux can be taken as a fractional $\operatorname{order}^{(26)}$ or as the integer order, ${ }^{(35)}$ resulting in different forms of fADEs.

First, we take the first-order conservation of mass, in which the diffusive flux is fractional order and also non-local. ${ }^{(35)}$ The fADE is of the form

$$
\frac{\partial C}{\partial t}=-\frac{\partial}{\partial x}[V(x) C]+\frac{\partial}{\partial x}\left[D(x) \frac{\partial^{\alpha-1} C}{\partial x^{\alpha-1}}\right] .
$$

Second, we take an alternative formulation using a fractional-order conservation of mass, obtained by replacing the integer order divergence in the usual formulation by a fractional analogue, ${ }^{(26)}$ to the local diffusive flux. The resultant fADE is

$$
\frac{\partial C}{\partial t}=-\frac{\partial}{\partial x}[V(x) C]+\frac{\partial^{\alpha-1}}{\partial x^{\alpha-1}}\left[D(x) \frac{\partial C}{\partial x}\right],
$$

where we keep the variation of flux caused by advection. Note that both (4) and (5) are equivalent in the case where $D(x)$ is a constant independent of the spatial variable $x$. It is only in the variable coefficient case where we must distinguish between the two cases (4) and (5).

The dispersion term in the fADE (5) can also be derived by taking the adjoint of the dispersive flux in the fADE (4) (Appendix A1):

$$
\left\{\frac{\partial}{\partial x}\left[D(x) \frac{\partial^{\alpha-1} C}{\partial x^{\alpha-1}}\right]\right\}^{*}=-\frac{\partial^{\alpha-1}}{\partial(-x)^{\alpha-1}}\left[D(x) \frac{\partial C}{\partial x}\right],
$$

although the adjoint of the advection term gives a different form from (5). The superscript $*$ in the above equation denotes the adjoint operator. Later in this paper we will show that (5) relates to a Markov process, whereas we have not been able to relate the general Eq. (4) to any Markov process. To distinguish the two fADEs, we denote the first version (4) as the FF-ADE since it contains a fractional flux, and the second (5) as the FD-ADE since it uses a fractional divergence.

\section{THE PARTICLE TRACKING SOLUTION OF FADE}

In this section, we explore the particle tracking algorithm for each of the two fADEs containing space-dependent parameters. Although the variability of mean water velocity does not change the algorithm of particle tracking discussed in the following, we consider the spatial variability of velocity too. For the purposes of comparison and validation, we also consider the case of spatially constant parameters. 


\subsection{The fADE with Spatially Constant $V$ and $D$}

When the groundwater velocity $V$ and the dispersion coefficient $D$ are space independent, both forms of the fADE (i.e., (4) and (5)) simplify to

$$
\frac{\partial C}{\partial t}=-V \frac{\partial C}{\partial x}+D \frac{\partial^{\alpha} C}{\partial x^{\alpha}},
$$

where $V$ and $D$ can be constant or functions of time.

To simulate the contaminant transport described by the fADE (7), we generate the following Markov process

$$
d X(t)=V d t+B d L_{\alpha}(t)
$$

where the random noise $d L_{\alpha}(t)$ can be calculated by

$$
d L_{\alpha}(t)=(d t)^{1 / \alpha} \cdot S_{\alpha}(\beta=1, \sigma=1, \mu=0),
$$

where $S_{\alpha}(\beta=1, \sigma=1, \mu=0)$ represents a maximally positively-skewed, standard, zero-mean $\alpha$-stable variable (see Ref. 33) for the definition of a standard $\alpha$-stable, see also Appendix A1).

Following Meerschaert et al. ${ }^{(24)}$ (see also Appendix A1), it is easy to verify that the correspondent density of particle numbers, $P$, satisfies the following forward equation

$$
\frac{\partial P}{\partial t}=-V \frac{\partial P}{\partial x}+\bar{D} B^{\alpha} \frac{\partial^{\alpha} P}{\partial x^{\alpha}}
$$

with $\bar{D}=-1 / \cos (\pi \alpha / 2)$. The particle number density $P$ in the above forward equation is equivalent to the solute concentration $C$ in (7). Therefore, the contaminant transport described by the fADE (7) can be solved by simulating the particle movement based on the Langevin equation (8) with $\bar{D} B^{\alpha}=D$. We note that this fADE (7) is the forward equation of the Markov process (8) or, equivalently, the associated abstract Cauchy problem considered by Baeumer and Meerschaert. ${ }^{(1)}$ Also note when $D$ is constant (no matter the property of $V$ ), the dynamics of solute caused by a fractional dispersion of order $\alpha$ can always be simulated by random-walking particles with random motions following an $\alpha$-order Lévy noise (see also Ref. 6).

\subsection{The FD-ADE (5) with Space-Dependent $V$ and $D$}

To simulate the contaminant transport with space dependent velocity and dispersion coefficients as described by the FD-ADE (5), we take adjoints as in Appendix A1 to obtain the corresponding backward equation

$$
\frac{\partial P}{\partial t}=V(x) \frac{\partial P}{\partial x}+\frac{\partial}{\partial(-x)}\left[D(x) \frac{\partial^{\alpha-1} P}{\partial(-x)^{\alpha-1}}\right],
$$


and rewrite this in the form

$$
\frac{\partial P}{\partial t}=V \frac{\partial P}{\partial x}+\bar{D}_{1} B_{1}^{\alpha} \frac{\partial^{\alpha} P}{\partial(-x)^{\alpha}}+\bar{D}_{2} B_{2}^{\alpha-1} \frac{\partial^{\alpha-1} P}{\partial(-x)^{\alpha-1}},
$$

where $\bar{D}_{1}=-1 / \cos (\pi \alpha / 2)$ and $\bar{D}_{1} B_{1}^{\alpha}=D$, while $\bar{D}_{2}=-1 / \cos (\pi(\alpha-1) / 2)$ and $\bar{D}_{2} B_{2}^{\alpha-1}=-\partial D / \partial x$. Following the same argument as in Appendix A1, we conclude that (10) is the backward equation for the Markov process

$$
d X(t)=V d t+B_{1} d L_{\alpha}(t)+B_{2} d L_{\alpha-1}(t),
$$

where the random noise $d L_{\alpha}(t)$ is the same as (9), and $d L_{\alpha-1}(t)$ is the corresponding Lévy stable noise with the index $\alpha-1$. Since a Lévy motion is restricted to $0<\alpha \leq 2$, simulation of the space-variable fADE by this method is limited to $1<\alpha \leq 2$. Also note that the dispersion coefficient $D$ is assumed to vary continuously in space in this study, since we assume that its derivative exists. For the case where $D$ has jumps, the dispersion-gradient term in (10) and (11) does not exist and the significant influence of heterogeneity interfaces on the drift of particles needs to be accounted for by additional terms in the Markov processes. Discontinuities of $D$ and other transport properties such as porosity commonly arise at abrupt contacts between geological materials (i.e., in composite porous media). The study of the smooth parameter case can provide significant insights for the discrete parameter case, and we will discuss the latter in a future paper.

\subsection{The FF-ADE (4) with Space-Dependent $V$ and $D$}

The Markov process approach may not be applicable to simulating the solute transport process obeying the FF-ADE (4) with a general form of dispersion coefficient $D$. To illustrate, if (4) were to represent the forward equation for some Markov process, the backward equation would have the form

$$
\frac{\partial P}{\partial t}=V(x) \frac{\partial P}{\partial x}-\frac{\partial^{\alpha-1}}{\partial(-x)^{\alpha-1}}\left[D(x) \frac{\partial P}{\partial x}\right]
$$

and the last term on the right expands into an infinite series of fractional derivatives and integrals using the fractional Leibniz rule: ${ }^{(30)}$

$$
\frac{\partial^{\alpha-1}}{\partial(-x)^{\alpha-1}}\left[D(x) \frac{\partial P}{\partial x}\right]=\sum_{n=0}^{\infty}\left(\begin{array}{c}
\alpha-1 \\
n
\end{array}\right) \frac{\partial^{n} D(x)}{\partial(-x)^{n}} \frac{\partial^{\alpha-n} P}{\partial(-x)^{\alpha-n}},
$$

where $\left(\begin{array}{l}\alpha \\ n\end{array}\right)=\frac{\Gamma(1+\alpha)}{\Gamma(1+\alpha-n) n !}$. We are not aware of any Markov process whose generator involves fractional integrals, hence we are not able to develop particle tracking codes for this equation in the case of a general $D(x)$ coefficient.

The application of a non-Markovian process is beyond the topic of this study. However, the Markov process is still applicable for specific cases; for example, 
when $V$ and $D$ vary linearly in space. For the remainder of this section, we assume that the water velocity and the dispersion coefficient are of the forms

$$
\begin{gathered}
V(x)=\eta x, \quad \eta>0, \\
D(x)=\alpha_{L} \quad V(x),
\end{gathered}
$$

where $\alpha_{L}\left[L^{\alpha-1}\right]$ is a constant dispersivity. This situation is thought to arise, for example, in an aquifer that grows thinner at a linear rate or receives uniform recharge from the surface. Now the infinite series in the fractional Leibnitz rule terminates and the backward equation is

$$
\frac{\partial P}{\partial t}=V(x) \frac{\partial P}{\partial x}+D \frac{\partial^{\alpha} P}{\partial(-x)^{\alpha}}-(\alpha-1) \frac{\partial D}{\partial x} \frac{\partial^{\alpha-1} P}{\partial(-x)^{\alpha-1}},
$$

which we rewrite in the form

$$
\frac{\partial P}{\partial t}=V \frac{\partial P}{\partial x}+\bar{D}_{1} B_{1}^{\alpha} \frac{\partial^{\alpha} P}{\partial(-x)^{\alpha}}+\bar{D}_{2} \varepsilon_{2}^{\alpha} \frac{\partial^{\alpha-1} P}{\partial(-x)^{\alpha-1}},
$$

where $\bar{D}_{1}=-1 / \cos (\pi \alpha / 2)$ and $\bar{D}_{1} B_{1}^{\alpha}=D$, while $\bar{D}_{2}=-1 / \cos (\pi(\alpha-1) / 2)$ and $\bar{D}_{2} \varepsilon_{2}^{\alpha-1}=-(\alpha-1) \partial D / \partial x$. Following the same argument as in Appendix A1, we conclude that (13) is the backward equation for the Markov process

$$
d X(t)=V d t+B_{1} d L_{\alpha}(t)+\varepsilon_{2} d L_{\alpha-1}(t),
$$

where the random noise $d L_{\alpha}(t)$ is the same as (9), and $d L_{\alpha-1}(t)$ is the corresponding Lévy stable noise with the index $\alpha-1$. Since a Lévy motion is restricted to $0<\alpha \leq 2$, simulation by this method is limited to $1<\alpha \leq 2$.

\section{RESULTS OF NUMERICAL EXPERIMENTS}

We test the random walk algorithms discussed above by comparing the random walk particle tracking results to the analytical solutions, or to other numerical solutions if analytical solutions are unavailable.

\subsection{Example 1: The fADE with Constant $V$ and $D$}

In this simplest case, we hold $V$ and $D$ constant in a one-dimensional infinite domain with the initial and boundary conditions

$$
\begin{gathered}
C(x, t=0)=\delta\left(x-x_{0}\right), \\
C(x= \pm \infty, t)=0,
\end{gathered}
$$

where $\delta\left(x-x_{0}\right)$ is a Dirac delta function representing an instantaneous point source located at $x_{0}$. The groundwater velocity $V$ is $0.24 \mathrm{~m} /$ day, and the dispersion 


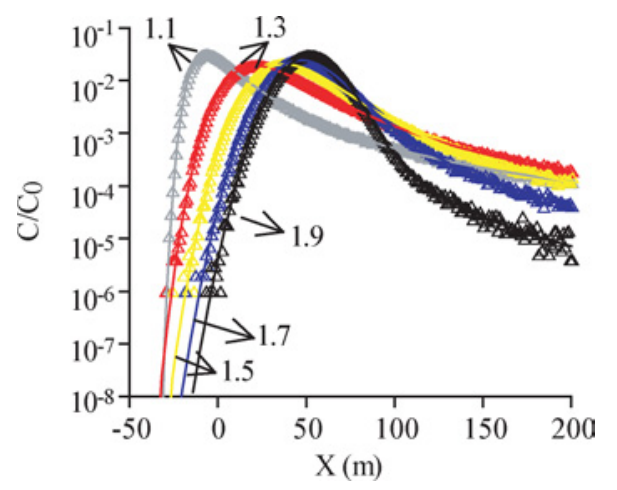

Fig. 1. Simulated tracer concentration by random walk particle tracking method (symbols) versus the analytical solutions (lines) at 244 days, for $1<\alpha<2$. The groundwater velocity $V$ is $0.24 \mathrm{~m} / \mathrm{day}$. The dispersion coefficient $D$ is $0.32 \mathrm{~m}^{\alpha} /$ day. The instantaneous point source is located at $x_{0}=0$. Color online.

coefficient $D$ is $0.32 \mathrm{~m}^{\alpha} /$ day. These values are the same order as those estimated by Benson et al. ${ }^{(3)}$ for the MADE site. The skewed stable variables were generated by the method proposed by Janicki and Weron. ${ }^{(14)} 1,000,000$ particles were released at $x_{0}=0$ and then tracked until the time of 244 days. We simulated the solute concentrations for $\alpha$ increasing from 1.1 to 1.9 with a step size 0.2 . The numerical solutions closely match the analytical solutions (Fig. 1). Simulation results show that the simulated particle number density (or the solute concentration) is not sensitive to the total number of time steps.

As shown by Fig. 1, the peak lags further behind for smaller $\alpha$ because the mean or center of mass is the same, and the leading tail is heavier, so the peak has to lag behind to compensate.

\subsection{Example 2: The FF-ADE (4) with a $D$ Increasing Linearly with Distance}

The random-walk algorithm (14) developed for solving the FF-ADE (4) with space-dependent $V$ and $D$ was validated by an extension of the implicit Euler finite difference method developed by Meerschaert and Tadjeran ${ }^{(21)}$ (Appendix A2).

Assume $\alpha=1.6$ and that $V$ and $D$ are simple linear functions of space: $V=$ $0.01 x \mathrm{~m} / \mathrm{yr}$, and $D=0.1 \mathrm{~V}=0.001 \times \mathrm{m}^{1.6} / \mathrm{yr}$. The simulated solute concentrations using the random walk algorithm (14) generally match the numerical solutions using the implicit Euler finite difference method (Fig. 2), implying the reliability of the Markov process (14). Figure 2(a) also shows that the simulated particle number density is not sensitive to the total number of time steps, which is consistent with 

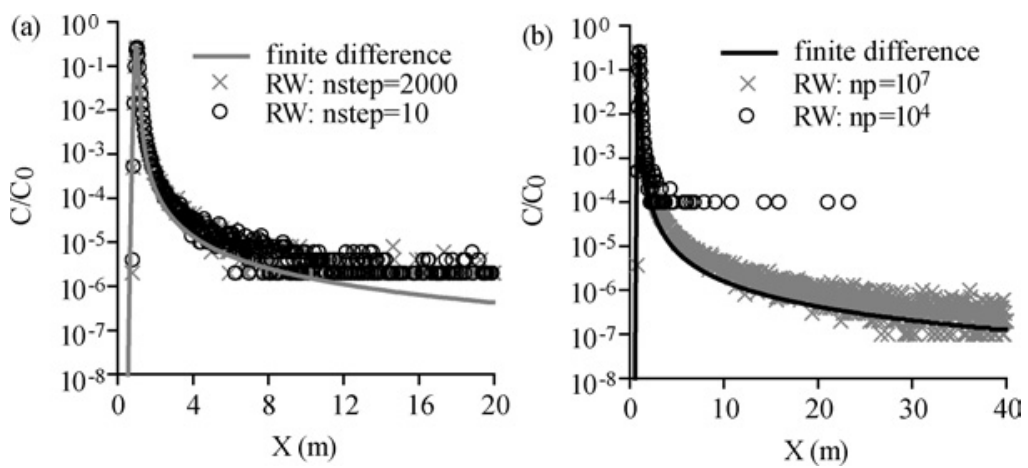

Fig. 2. Effects of the time step size (a) and the number of particles (b) on the simulated concentrations at $T=50$ yrs using random walk particle tracking method. "finite difference" denotes the numerical solutions of the implicit Euler finite difference method, and "RW" denotes the random walk method. In (a), "nstep" represents the number of total time steps. The 10 and 2000 of nstep denote the time step is 5 yrs and $0.025 \mathrm{yrs}$, respectively. In (b), "np" represents the number of particles. The groundwater velocity $V$ is $0.01 x \mathrm{~m} / \mathrm{yr}$, and the dispersion coefficient $D$ is $0.001 x \mathrm{~m}^{1.6} / \mathrm{yr}$.

the result discussed in Section 4.1. However, the solution along the leading edge of the plume, where concentrations are lowest, is sensitive to the total number of particles used in the random walk simulations.

Extending these results, a future study may apply a Markov process with constant diffusion coefficient to fitting the solute transport obeying the FF-ADE (4) with a general form of dispersion coefficient. The three terms of particle dynamics in (14) can be adjusted to characterize the influences of spatial-dependent water velocity and dispersion on solute transport, and to investigate the relative importance of the truncation of terms in the sums in the infinite series.

\subsection{Example 3: The FD-ADE (5) with Space-Dependent $V$ and $D$}

The random-walk algorithm (11) developed for solving the FD-ADE (5) with space-dependent $V$ and $D$ was tested for two cases. In the first case, we checked the algorithm indirectly by selecting a specific $\alpha$. If $\alpha$ is very close to 2, then the FD-ADE (5) should have similar solutions as a second-order ADE. It implies that the Markov process (11) should result in a similar particle number density as the Markov process used for the second-order ADE.

The second-order ADE with space-dependent $V$ and $D$ can be solved by the following Markov process ${ }^{(18)}$

$$
d X(t) \approx A(x) d t+B(x) d \omega(t)
$$




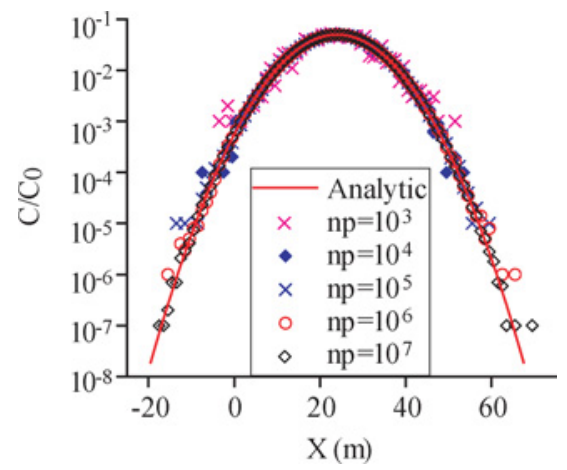

Fig. 3. Simulated tracer concentrations for the Gaussian $(\alpha=2)$ case, using the random walk particle tracking method (symbols) versus the analytic solution (line). The groundwater velocity $V$ is 0.24 $\mathrm{m} /$ day. The dispersion coefficient $D$ is $0.32 \mathrm{~m}^{2} /$ day. The instantaneous point source is located at $x_{0}=0$. The time is 100 days. Color online.

The drift $A$ and the diffusion $B$ are defined by water velocity $V$ and dispersion coefficient $D$ [also see, Ref. 17 Eqs. (10) and (11)]:

$$
\begin{gathered}
A=V+\partial D / \partial x, \\
B=\sqrt{2 D} .
\end{gathered}
$$

The $d \omega(t)\left[T^{1 / 2}\right]$ in (15) represents a Wiener process, and it can be expressed by

$$
d \omega(t)=\xi \sqrt{d t},
$$

where $\xi$ denotes independent normally distributed random variables with zero mean and unit variance.

We first tested the Markov process (15) using the same constant $V$ and $D$ as in Example 1. The symmetric distribution of the simulated concentrations matches the analytical solutions, and the accuracy of the random walk method depends on the number of particles (Fig. 3). Then we solved the second-order ADE with spacedependent $V$ and $D$. Assuming $V=0.1 x \mathrm{~m} /$ day and $D=0.06 x \mathrm{~m}^{2} /$ day for the second-order ADE, we solved the solute concentration using the Markov process (15). A Dirac pulse of contaminants is injected at $x_{0}=25 \mathrm{~m}$. A one-dimensional semi-infinite domain $(x \geq 0)$ is considered, and the boundary concentration at both ends remains zero. At the upstream boundary $x=0, V=D=0$. Also by assuming $V=0.1 x \mathrm{~m} /$ day, $D=0.06 x \mathrm{~m}^{1.999} /$ day, and $\alpha=1.999$ for the fADE (5), and using the same initial and boundary conditions, we solved the solute concentration using the Markov process (11). These two numerical solutions are essentially identical (Fig. 4a).

In the second case, we tested the applicability of the random-walk algorithm (11) for arbitrary $1<\alpha<2$ by comparing the particle tracking results to another 

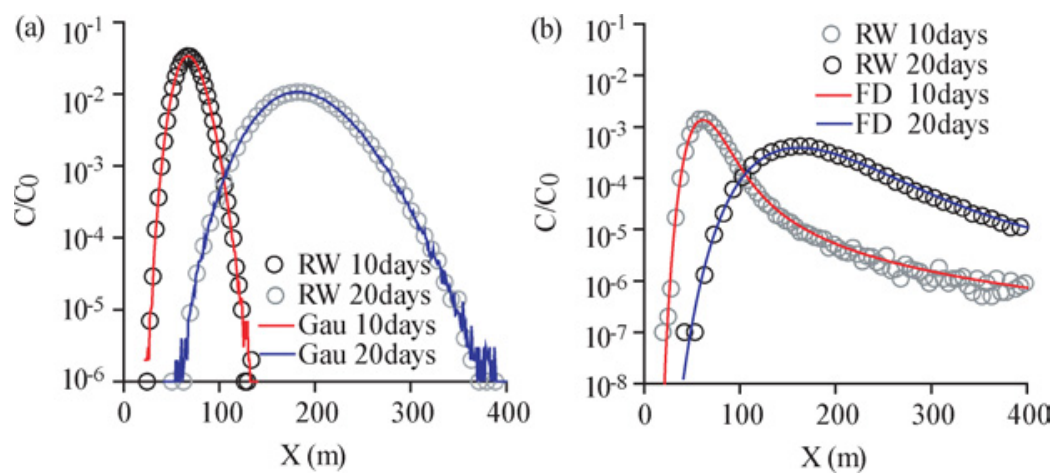

Fig. 4. Simulated concentration profiles for the linearly varying coefficients $V=0.1 x \mathrm{~m} /$ day and $D=0.06 x \mathrm{~m}^{\alpha} /$ day. The instantaneous point source is located at $x_{0}=25 \mathrm{~m}$. (a) The solid line ("Gau") represents the numerical solutions of Gaussian case $(\alpha=2)$, and the circles ("RW") represent the numerical solutions of random walk particle tracking method $(\alpha=1.999)$. The time is 10 and 20 days, respectively. (b) The solid line ("FD") represents the numerical solutions of the implicit Euler finite difference method ( $\alpha=1.7)$, and the circle ("RW") represents the numerical solutions of random walk particle tracking method $(\alpha=1.7)$. The times are 10 and 20 days, respectively. Color online.

numerical solution similar to that discussed in Appendix A2, which is an extension of the implicit Euler finite difference method developed by Meerschaert and Tadjeran. ${ }^{(21)}$ The $V$ and $D$ used in the first case remain the same in the second case, for the purpose of comparison. In this case the FD-ADE (5) simplifies to

$$
\begin{aligned}
\frac{\partial C}{\partial t} & =-\frac{\partial}{\partial x}[V(x) C]+\frac{\partial^{\alpha-1}}{\partial x^{\alpha-1}}\left[D(X) \frac{\partial C}{\partial x}\right] \\
& =-\frac{\partial}{\partial x}(V C)+D(x) \frac{\partial^{\alpha-1}}{\partial x^{\alpha-1}}\left(\frac{\partial C}{\partial x}\right)+(\alpha-1) \frac{\partial D(x)}{\partial x} \frac{\partial^{\alpha-2}}{\partial x^{\alpha-2}}\left(\frac{\partial C}{\partial x}\right) \\
& =-\frac{\partial}{\partial x}[V(x) C]+D(x) \frac{\partial^{\alpha} C}{\partial x^{\alpha}}+(\alpha-1) \frac{\partial D(x)}{\partial x} \frac{\partial^{\alpha-1} C}{\partial x^{\alpha-1}} .
\end{aligned}
$$

In the last step we replace the fractional integral of derivatives with derivatives of the fractional integral (see also, Ref. 29, p. 59, Theorem 2), and use the condition that the solute concentration at the left boundary is zero. Following the same process as in Appendix A2, we find that the above fADE can be solved numerically by the implicit Euler finite difference method, and the numerical solutions will be stable as long as the spatial grid size $\Delta x$ satisfies

$$
\Delta x \leq\left[\frac{V_{i}-V_{i-1}}{2(\alpha-1)\left(D_{i}-D_{i-1}\right)}\right]^{1 / 1-\alpha},
$$

where the subscript $i$ denotes the spatial grid. The two different numerical methods (Lagrangian versus Eulerian) give essentially identical solutions (Fig. 4(b)). This 
equation is a sufficient condition for the variable velocity model (5), but other conditions apply for the constant velocity or pure diffusion model. Proposition 2.1 in Ref. 20 shows that for an explicit Euler scheme, stability requires that $\Delta t / \Delta x^{\alpha}$ is bounded. This also agrees with the condition in Gorenflo et al. (see Eq. (2.7) in Ref. 9, Eq. (23) in Ref. 11, and Eq. (33) in Ref. 10).

Note that the second Lévy motion in (11) serves to describe the influence of the spatial variation of dispersion coefficients on solute transport. The variation of dispersion coefficients causes the particle to have additional movement over some time increment, which can be expressed as a scaled Lévy motion with an order of $\alpha-1$. The scaling factor depends on both the gradient of dispersion coefficient and the fractional-order of the general dispersion in the fADE. When the fADE (5) reduces to the 2nd-order ADE, the drift displacement of particles defined by (11) reduces to $(V+\partial D / \partial x) d t$, since $d L_{\alpha-1}(t) \propto(d t)^{\alpha-1}$, as expected in (16). Also note again that the $\alpha$-order Lévy motion in (11) has a different scaling factor $\left(D^{1 / \alpha}\right)$ than the Brownian motion in $(15)(\sqrt{2 D})$, since a standard stable with $\alpha=2$ is not standard normal.

\section{DISCUSSION}

The Markovian particle jump process may not be exactly the same as the solute transport process. In other words, the forward equation may be different from the fADE. First, the forward equation provides a Lagrangian description of the dynamics of random-walking particles. The fADE, however, provides an Eulerian description of solute fluxes. The translation from one to the other typically requires an accounting of the spatial versus temporal variability of transport parameters. Second, the form of the forward equation varies with the underlying Markov process. Third, as also indicated by numerous other numerical experiments for the Gaussian case (i.e., Refs. 15, 17, 38), the particles and the solutes have different forcing factors if the dispersion coefficient varies with space. When the dispersion coefficient does not change with space, the forward equation has the same form as the FD-ADE, but it is still different from the FF-ADE. There is no reason to treat them equally; for example, it may not be reasonable to explore the form of advection-dispersion equation using random walkers (such as continuous time random walks, e.g., Ref. 34) without distinguishing between the forward equation and ADE.

In the FD-ADE (5), the variation of diffusive flux within a representative elementary volume (REV) is caused by the diffusive fluxes at all upgradient zones. In the FF-ADE (4), the variation of diffusive flux within the REV is caused by the concentrations at all upgradient zones. For the case that the dispersion coefficient $D$ increases linearly with distance, the difference between the fADEs can be reflected by the different $(\alpha-1)$-order Lévy noise for particle movements described by Markov processes (11) and (14). In the FD-ADE, the particles have a relatively 
larger movements caused by the ( $\alpha-1)$-order Lévy noise, implying larger influence caused by the spatial variations of dispersion coefficients. Therefore, the main difference of the two fADEs is that the particle movements contain more memory of the dispersion coefficient in the FD-ADE than in the FF-ADE.

In addition, when $\alpha$ increases to 2, the dispersive flux in the FD-ADE is similar to that in the FF-ADE, and the equivalent Markov processes also have a similar form. Thus all Markov processes discussed in this study can be simplified to the traditional particle tracking algorithm when the order $\alpha=2$.

This study emphasizes the difficulties encountered when solving a fractional dispersion equation. However, the equations seek to represent the micro-scale differential advective fluxes with a simpler dispersive term, hence the numerical aspects of solving for velocity on a very fine grid are eliminated. It remains to be shown whether solving a coarse-grid fractional equation is more computationally efficient than solving a classical ADE on a very fine grid. In multiple dimensions, the mean flow fields can be highly curved, and the Lévy motion skewness is represented by a probability measure on the unit sphere (see some numerical aspects explored by Roop ${ }^{(32)}$ ).

Sokolov and Metzler ${ }^{(36)}$ demonstrated that the space-fractional diffusion equation is not uniquely connected to a trajectory, so that the fADE solutions do not contain all the relevant information about the trajectories. Hence it is possible that alternative particle tracking schemes also exist, as different stochastic models may have the same governing equations for the particle tracking.

In a heterogeneous porous medium, it may also be useful to consider a fADE in which the order of the fractional derivative is a function of the spatial variable. A related model with a space-varying order of a fractional time derivative was recently considered by Chechkin et al. ${ }^{(5)}$ for a problem in fractional kinetics. That extension is beyond the scope of this paper, and will be investigated in a future study.

\section{CONCLUSIONS}

1. The fADE of order $0<\alpha \leq 2$, with constant or time-dependent coefficients, may be solved by using a Markov process with a Lévy noise of order $\alpha$ and a simple drift. The particle drift equals the advection of solutes, and the Lévy noise of particle displacements represents the random motion of solutes caused by local differential advection.

2. When the dispersion coefficient $D$ varies in space, the FF-ADE (4) of order $1<\alpha \leq 2$ cannot be solved by Markov processes, except for specific $D$ such as a linear function of space.

3. When $D$ varies continuously in space and the solute concentration at the upstream boundary remains zero, the FD-ADE (5) of order $1<\alpha \leq 2$ can be solved by simulating a Markov process with a simple drift, an $\alpha$-stable 
Lévy noise and an additional term with the dispersion gradient and an additional Lévy noise of order $\alpha-1$.

\section{A.1. THE FORWARD EQUATION DERIVED BY THE ADJOINT METHOD}

Let $x(t)$ be a Markov process with transition density $q_{t}(x, y)$, denoting the likelihood of $x(t)=y$ given $x(0)=x$. If we let

$$
u(t, x)=\int q_{t}(x, y) u_{0}(y) d y,
$$

then $u(t, x)$ is the expected value of $u_{0}(x(t))$ given that $x(0)=x$. If we define

$$
T_{t} u_{0}(x)=\int q_{t}(x, y) u_{0}(y) d y,
$$

then $T_{t}$ is a semigroup ${ }^{(8)}$ with generator $L$ defined as

$$
L u_{0}(x)=\lim _{t \rightarrow 0+} \frac{T_{t} u_{0}(x)-T_{0} u_{0}(x)}{t-0}=\lim _{t \rightarrow 0+} \frac{T_{t} u_{0}(x)-u_{0}(x)}{t},
$$

and then the function

$$
u(t, x)=T_{t} u_{0}(x)
$$

solves an abstract Cauchy problem

$$
\frac{\partial u}{\partial t}=L u, \quad u(x, 0)=u_{0}(x) .
$$

This is also called the backward equation, since it maps probabilities backward in time. The non-linear Langevin equation (1):

$$
d X(t)=A(x, t) d t+B(x, t) d L(t)
$$

defines a Markov process with the following generator (Ref. 7, p. 379, Theorem 3.3; and Ref. 37)

$$
\begin{aligned}
& L u=A(x, t) \frac{\partial u}{\partial x}+\int\left[u(x+y)-u(x)-\frac{y}{1+y^{2}} \frac{\partial u}{\partial x}\right] \\
& B(x, t)^{\alpha} \phi(d y)
\end{aligned}
$$

where $\phi(d y)=C p \alpha y^{-\alpha-1} d y$ for $y>0$ and $\phi(d y)=C p \alpha|y|^{-\alpha-1} d y$ for $y<0$ with $C=(1-\alpha) /[\Gamma(2-\alpha) \cos (\pi \alpha / 2)]$ is the Lévy measure of a standard stable law (see, e.g., Ref. 23, Theorem 7.3.5) and $B(x, t)$ is continuous. Note that for $1<\alpha<2$ we have ${ }^{(1)}$

$$
\frac{d^{\alpha} f(x)}{d x^{\alpha}}=\frac{\alpha-1}{\Gamma(2-\alpha)} \int_{0}^{\infty}\left[f(x-y)-f(x)+y f^{\prime}(x)\right] \alpha y^{-1-\alpha} d y
$$




$$
\frac{d^{\alpha} f(x)}{d(-x)^{\alpha}}=\frac{\alpha-1}{\Gamma(2-\alpha)} \int_{0}^{\infty}\left[f(x+y)-f(x)-y f^{\prime}(x)\right] \alpha y^{-1-\alpha} d y
$$

Then we can take (after a little rearrangement) the generator to be of the form

$$
L u=A(x, t) \frac{\partial u}{\partial x}+\bar{D} q B(x, t)^{\alpha} \frac{\partial^{\alpha} u}{\partial x^{\alpha}}+\bar{D} p B(x, t)^{\alpha} \frac{\partial^{\alpha} u}{\partial(-x)^{\alpha}}
$$

with $\bar{D}=-1 / \cos (\pi \alpha / 2)$ compare Ref. 24 and then $u(t, x)$ solves the backward equation

$$
\frac{\partial u}{\partial t}=A \frac{\partial u}{\partial x}+\bar{D} q B^{\alpha} \frac{\partial^{\alpha} u}{\partial x^{\alpha}}+\bar{D} p B^{\alpha} \frac{\partial^{\alpha} u}{\partial(-x)^{\alpha}}
$$

where $A, B$ may depend on both $x$ and $t$, and $p=(1+\beta) / 2$ and $q=(1-\beta) / 2$ relating back to the skewness parameter of the stable law.

Let $v(s, y)$ be the density of $x(s)=y$ given that $x(0)$ has density $v_{0}(x)$, then

$$
v(s, y)=\int q_{s}(x, y) v_{0}(x) d x .
$$

We will derive the forward equation by extending the classical approach (Ref. 8, p. 338) to the fractional case. Let

$$
\begin{aligned}
H(s, t) & =\int v(s, y) u(t, y) d y \\
& =\iint q_{s}(x, y) v_{0}(x) d x \int q_{t}(y, z) u_{0}(z) d z d y \\
& =\iint\left(\int q_{s}(x, y) q_{t}(y, z) d y\right) v_{0}(x) u_{0}(z) d x d z \\
& =\iint q_{s+t}(x, z) v_{0}(x) u_{0}(z) d x d z
\end{aligned}
$$

so $H(s, t)=G(s+t)$ only depends on $s+t$. Then

$$
\frac{\partial H}{\partial s}=\frac{\partial H}{\partial t}
$$

and so

$$
\frac{\partial}{\partial t} \int v(s, y) u(t, y) d y=\frac{\partial}{\partial s} \int v(s, y) u(t, y) d y
$$

which is equal to

$$
\int v(s, y) \frac{\partial}{\partial t} u(t, y) d y=\int u(t, y) \frac{\partial}{\partial s} v(s, y) d y .
$$


Now substitute the backward Eq. (A.3) in the left-hand side of the above equation to get

$$
\int v(s, y) L u(t, y) d y=\int u(t, y) \frac{\partial}{\partial s} v(s, y) d y .
$$

Substitute (A.2) to get

$$
\begin{aligned}
\int v(s, y)\left[A \frac{\partial u(t, y)}{\partial y}\right. & \left.+\bar{D} q B^{\alpha} \frac{\partial^{\alpha} u(t, y)}{\partial y^{\alpha}}+\bar{D} p B^{\alpha} \frac{\partial^{\alpha} u(t, y)}{\partial(-y)^{\alpha}}\right] d y \\
& =\int u(t, y)\left[\frac{\partial}{\partial s} v(s, y)\right] d y
\end{aligned}
$$

which can be shown equivalent to

$$
\begin{gathered}
\int u(t, y)\left[-\frac{\partial}{\partial y}(A v(s, y))+\bar{D} q \frac{\partial^{\alpha}}{\partial(-y)^{\alpha}}\left(B^{\alpha} v(s, y)\right)+\bar{D} p \frac{\partial^{\alpha}}{\partial y^{\alpha}}\left(B^{\alpha} v(s, y)\right)\right] d y \\
=\int u(t, y)\left[\frac{\partial}{\partial s} v(s, y)\right] d y .
\end{gathered}
$$

Letting $t \rightarrow 0, u(t, y) \rightarrow u_{0}(y)$, an arbitrary function, so the forward equation follows:

$$
\frac{\partial}{\partial s} v(s, x)=-\frac{\partial}{\partial x}(A v(s, x))+\bar{D} q \frac{\partial^{\alpha}}{\partial(-x)^{\alpha}}\left(B^{\alpha} v(s, x)\right)+\bar{D} p \frac{\partial^{\alpha}}{\partial x^{\alpha}}\left(B^{\alpha} v(s, x)\right) \text {. }
$$

Clouds of particles that follow the Langevin equation (1) can therefore be tracked to solve the forward Eq. (A.5).

To see why the two integral forms are equivalent, consider each of the three terms in square brackets separately. For the first term, we get immediately via integration by parts that

$$
\int v(s, y) A(y, t) \frac{\partial u(t, y)}{\partial y} d y=-\int \frac{\partial}{\partial y}[v(s, y) A(y, t)] u(t, y) d y .
$$

For the second term, use an alternate formula for the positive fractional derivative $^{(22)}$ to get

$$
\begin{aligned}
\int v(s, y)[ & \left.\bar{D} q B(y, t)^{\alpha} \frac{\partial^{\alpha} u(t, y)}{\partial y^{\alpha}}\right] d y=\int v(s, y) \\
& {\left[\bar{D} q B(y, t)^{\alpha} \frac{1}{\Gamma(2-\alpha)} \int_{0}^{\infty} \frac{\partial^{2} u(t, y-z)}{\partial y^{2}} z^{1-\alpha} d z\right] d y . }
\end{aligned}
$$


Switch the order of integration and substitute $x=y-z$ in the integral on the right to get

$$
\int_{0}^{\infty} \bar{D} q \frac{1}{\Gamma(2-\alpha)} z^{1-\alpha}\left[\int v(s, x+z) B(x+z, t)^{\alpha} \frac{\partial^{2} u(t, x)}{\partial y^{2}} d x\right] d z .
$$

Finally, integrate by parts twice in the inner integral to get

$$
\begin{aligned}
& \int_{0}^{\infty} \bar{D} q \frac{1}{\Gamma(2-\alpha)} z^{1-\alpha}\left[\int \frac{\partial^{2}}{\partial x^{2}}\left\{v(s, x+z) B(x+z, t)^{\alpha}\right\} u(t, x) d x\right] d z \\
& \quad=\int u(t, x)\left[\bar{D} q \frac{1}{\Gamma(2-\alpha)} \int_{0}^{\infty} \frac{\partial^{2}}{\partial x^{2}}\left\{v(s, x+z) B(x+z, t)^{\alpha}\right\} z^{1-\alpha} d z\right] d x \\
& \quad=\int u(t, x) \bar{D} q \frac{\partial^{\alpha}}{\partial(-x)^{\alpha}}\left[v(s, x) B(x, t)^{\alpha}\right] d x .
\end{aligned}
$$

In the last step we use an alternate formula for the negative fractional derivative. ${ }^{(22)}$ The third term is similar to the second, and is left to the reader. An additional term with $0<\alpha<1$ in the Langevin equation (1) leads to additional additive terms in the forward equation. An argument similar to the one detailed above shows that these additional terms have the same form as the $1<\alpha<2$ term in (A.5), but note that $\bar{D}=-1 / \cos (\pi \alpha / 2)$ is negative for $0<\alpha<1$ and positive for $1<\alpha<2$.

\section{A.2. SOLVING THE FADE WITH SPACE-DEPENDENT PARAMETERS BY THE IMPLICIT EULER FINITE DIFFERENCE METHOD}

The fractional advection-dispersion equation with space-dependent coefficients is:

$$
\frac{\partial C(x, t)}{\partial t}=-\frac{\partial}{\partial x}[V(x) C(x, t)]+\frac{\partial}{\partial x}\left[D(x) \frac{\partial^{\alpha-1} C(x, t)}{\partial x^{\alpha-1}}\right]+S(x, t),
$$

where $0<\alpha-1<1$, and $S(x, t)$ represents the source/sink term. For the simplicity, we assume that the velocity $V(x)$ and $D(x)$ are functions in space, but not in time. This is corresponding to the case of steady-state flow. We also assume that $V(x)$ are positive, and $V(x)$ and $D(x)$ are monotone increasing with $x$, to keep consistent with Section 3.2.

Using the implicit Euler method, the above fADE can be solved as

$$
\begin{aligned}
\frac{C_{i}^{n+1}-C_{i}^{n}}{\Delta t}= & -V_{i} \frac{C_{i}^{n+1}-C_{i-1}^{n+1}}{\Delta x}-C_{i}^{n+1} \frac{V_{i}-V_{i-1}}{\Delta x}+D_{i} \sum_{k=0}^{i+1} \frac{1}{h^{\alpha}} g_{k} C_{i-k+1}^{n+1} \\
& +\frac{D_{i}-D_{i-1}}{\Delta x} \sum_{k=0}^{i} \frac{1}{h^{\alpha-1}} f_{k} C_{i-k}^{n+1}+S_{i}^{n+1}
\end{aligned}
$$


where $g_{k}$ is the Grünwald weight for the $\alpha$-order fractional derivative, and $f_{k}$ is the Grünwald weight for the $(\alpha-1)$-order fractional derivative. ${ }^{(21)}$ Note that $h=\Delta x$ here.

Equation (A.9) can be built for every node, thus we can get the following equations:

$$
[F] C^{n+1}=C^{n}+\Delta t S^{n+1},
$$

where $[F]$ is a $(K+1) \times(K+1)$ coefficient matrix; $C^{n+1}, C^{n}$, and $S^{n+1}$ are $(K+1) \times 1$ matrix (i.e., a vector); and $K+1$ denotes the total number of nodes.

The entry in $[F]$ is

$$
F_{i, j}= \begin{cases}0, & \text { if } j>i+1 \\ -D_{i} \frac{\Delta t}{h^{\alpha}} g_{0}, & \text { if } j=i+1 \\ 1+V_{i} \frac{\Delta t}{h}+\Delta t \frac{V_{i}-V_{i-1}}{h}-D_{i} \frac{\Delta t}{h^{\alpha}} g_{1}-\frac{D_{i}-D_{i-1}}{\Delta x} \frac{\Delta t}{h^{\alpha-1}} f_{0}, & \text { if } j=i \\ -V_{i} \frac{\Delta t}{h}-D_{i} \frac{\Delta t}{h^{\alpha}} g_{2}-\frac{D_{i}-D_{i-1}}{h} \frac{\Delta t}{h^{\alpha-1}} f_{1}, & \text { if } j=i-1 \\ -D_{i} \frac{\Delta t}{h^{\alpha}} g_{i-j+1}-\frac{D_{i}-D_{i-1}}{h} \frac{\Delta t}{h^{\alpha-1}} f_{i-j}, & \text { if } j<i-1\end{cases}
$$

Choose $i$ so that $\left|x_{i}\right|=\max \left\{\left|x_{j}\right|: j=0,1, \ldots, K\right\}$, and then the eigenvalue of matrix $[F]$ is ${ }^{(21)}$

$$
\begin{aligned}
\lambda= & F_{i, j}+\sum_{j=0, j \neq i}^{K} F_{i, j} \frac{x_{j}}{x_{i}}=1+V_{i} \frac{\Delta t}{\Delta x}\left[1-\frac{x_{i-1}}{x_{i}}\right]+\Delta t \frac{V_{i}-V_{i-1}}{\Delta x} \\
& -D_{i} \frac{\Delta t}{h^{\alpha}}\left[g_{1}+\sum_{j=0, j \neq i}^{i+1} g_{i-j+1} \frac{x_{j}}{x_{i}}\right]-\frac{D_{i}-D_{i-1}}{\Delta x} \frac{\Delta t}{h^{\alpha-1}}\left[f_{0}+\sum_{j=0, j \neq i}^{i-1} f_{i-j} \frac{x_{j}}{x_{i}}\right] .
\end{aligned}
$$

Since the 2 nd term on the right hand site of (A.11) is non-negative, thus the eigenvalue $\lambda$ has real part exceeding 1 if:

$$
\begin{aligned}
\operatorname{Re}\left\{\Delta t \frac{V_{i}-V_{i-1}}{\Delta t}\right. & -D_{i} \frac{\Delta t}{h^{\alpha}}\left[g_{1}+\sum_{j=0, j \neq i}^{i+1} g_{i-j+1} \frac{x_{j}}{x_{i}}\right] \\
& \left.-\frac{D_{i}-D_{i-1}}{\Delta x} \frac{\Delta t}{h^{\alpha-1}}\left[f_{0}+\sum_{j=0, j \neq i}^{i-1} f_{i-j} \frac{x_{j}}{x_{i}}\right]\right\} \geq 0 .
\end{aligned}
$$

Now we look for the requirements of spatial and temporal step sizes, $\Delta x$ and $\Delta t$, in order to satisfy the inequality (A.12).

In a zero-shift Grünwald approximation for an $\alpha-1$ order $(0<\alpha-1<1)$ fractional derivative, the only positive term in the sequence of Grünwald weights is $f_{0}=1$. Note also that the weights sum to zero. Therefore, $0 \geq \sum_{j=0, j \neq i}^{i-1} f_{i-j} \geq$ 
$-f_{0}$ for any $i=1,2, \cdots$. Since $\left|x_{j} / x_{i}\right| \leq 1$ and $f_{j} \leq 0$ for $j=1,2, \cdots, K$, we get:

$$
1 \geq f_{0}+\sum_{j=0, j \neq i}^{i-1} f_{i-j}\left|\frac{x_{j}}{x_{i}}\right| \geq 0 .
$$

In a 1 -shift Grünwald approximation for an $\alpha$ order $(1<\alpha<2)$ fractional derivative, the only negative term in the sequence of Grünwald weights is $g_{1}=-\alpha$. Therefore, $\sum_{j=0, j \neq i}^{i+1} g_{i-j+1} \leq-g_{1}$ for any $i=0,1,2, \ldots$. Since $\left|x_{j} / x_{i}\right| \leq 1$ and $g_{j} \geq 0$ for $j=0,2, \ldots, K$, we get:

$$
-\alpha \leq g_{1}+\sum_{j=0, j \neq i}^{i+1} g_{i-j+1}\left|\frac{x_{j}}{x_{i}}\right| \leq 0 .
$$

Define $\omega=\sum_{j=0, j \neq i}^{i+1} g_{i-j+1} \frac{x_{j}}{x_{i}}$ and note the complex number $|\omega|<\alpha$. So $-D_{i} \frac{\Delta t}{h^{\alpha}}\left[g_{1}+\sum_{j=0, j \neq i}^{i+1} g_{i-j+1} \frac{x_{j}}{x_{i}}\right]=D_{i} \frac{\Delta t}{h^{\alpha}}[\alpha-\omega]$ has positive real part, since $\alpha-\omega$ lies in a circle $D:\{z \in C:|z-\alpha|<\alpha\}$ in the complex plane consisting of complex numbers that all have positive real part. Therefore, the inequality (A.12) will be satisfied if

$$
\operatorname{Re}\left\{\Delta t \frac{V_{i}-V_{i-1}}{\Delta t}-\frac{D_{i}-D_{i-1}}{\Delta x} \frac{\Delta t}{h^{\alpha-1}}\left[f_{0}+\sum_{j=0, j \neq i}^{i-1} f_{i-j} \frac{x_{j}}{x_{i}}\right]\right\} \geq 0 .
$$

Since $\frac{D_{i}-D_{i-1}}{\Delta x} \frac{\Delta t}{h^{\alpha-1}}$ in the above inequality are non-negative reals, we get the following relationship based on (A.13):

$$
-\frac{D_{i}-D_{i-1}}{\Delta x} \frac{\Delta t}{h^{\alpha-1}}\left[f_{0}+\sum_{j=o, j \neq i}^{i-1}\left|\frac{x_{j}}{x_{i}}\right|\right] \geq-\frac{D_{i}-D_{i-1}}{\Delta x} \frac{\Delta t}{h^{\alpha-1}} .
$$

Therefore the inequality (A.12) will be satisfied if

$$
\Delta t \frac{V_{i}-V_{i-1}}{\Delta x}-\frac{D_{i}-D_{i-1}}{\Delta x} \frac{\Delta t}{h^{\alpha-1}} \geq 0 .
$$

Define $\omega^{\prime}=\sum_{j=0, j \neq i}^{i-1} f_{i-j} \frac{x_{j}}{x_{i}}$ and note that $\left|\omega^{\prime}\right|<1$. Then we have $-\frac{D_{i}-D_{i-1}}{\Delta x} \frac{\Delta t}{h^{\alpha-1}}\left[f_{0}+\sum_{j=o, j \neq i}^{i-1}\left|\frac{x_{j}}{x_{i}}\right|\right]=-\frac{D_{i}-D_{i-1}}{\Delta x} \frac{\Delta t}{h^{\alpha-1}}\left[1+\omega^{\prime}\right]$, and since $\left|\omega^{\prime}\right|<1$ this implies that $\left|-\frac{D_{i}-D_{i-1}}{\Delta x} \frac{\Delta t}{h^{\alpha-1}}\left[1+\omega^{\prime}\right]\right| \leq 2\left[\frac{D_{i}-D_{i-1}}{\Delta x} \frac{\Delta t}{h^{\alpha-1}}\right]$ since $\left|1+\omega^{\prime}\right|<2$. Now (A.15) will follow as long as:

$$
\Delta t \frac{V_{i}-V_{i-1}}{\Delta x} \geq 2\left[\frac{D_{i}-D_{i-1}}{\Delta x} \frac{\Delta t}{h^{\alpha-1}}\right] .
$$


In the above inequality, $\Delta t>0$ and $\Delta x=h>0$. Based on the previous assumption (i.e., $V(x)$ and $D(x)$ increases with $x),\left(V_{i}-V_{i-1}\right)>0$ and $\left(D_{i}-D_{i-1}\right)>0$, we have:

$$
\Delta x \leq\left[\frac{1}{2} \frac{V_{i}-V_{i-1}}{D_{i}-D_{i-1}}\right]^{1 / 1-\alpha},
$$

which is the stability requirement of (A.9). In conclusion, the implicit Euler method is stable for any time step size $\Delta t$ as long as the spatial grid $\Delta x$ is sufficiently small.

\section{ACKNOWLEDGEMENTS}

This material is based upon work supported by the National Science Foundation under Grant Nos. DMS-0417869, DMS-0417972, DMS-0139927, DMS0139943.

\section{REFERENCES}

1. B. Baeumer and M. M. Meerschaert, Frac. Calc. Appl. Anal 4:481 (2001).

2. D. A. Benson, Ph.D. dissertation, University of Nevada at Reno, 1998 (unpublished).

3. D. A. Benson, R. Schumer, M. M. Meerschaert, and S. W. Wheatcraft, Transp. Por. Media 42:211 (2001).

4. D. A. Benson, S. W. Wheatcraft, and M. M. Meerschaert, Water Resour. Res. 36(6):1403 (2000).

5. A. V. Chechkin, R. Gorenflo and I. M. Sokolov, J. Phys. A 38:L679 (2005).

6. A. V. Chechkin, V. Y. Gonchar, J. Klafter, R. Metzler, and L. V. Tanatarov, J. Sta. Phys. 115:1505 (2004).

7. S. N. Ethier and T. G. Kurtz, Markov Processes: Characterization and Convergence, p. 380, John Wiley \& Sons, New York, 1986.

8. W. Feller, An introduction to Probability Theory and Its Applications, second edition, John Wiley \& Sons, New York, 1971.

9. R. Gorenflo and F. Mainardi, J. Anal. Appl. 18(2):231 (1999).

10. R. Gorenflo, A. Vivoli, and F. Mainardi, Nonlinear Dynamics 38:101 (2004).

11. R. Gorenflo, F. Mainardi, D. Moretti, and P. Paradisi, Nonlinear Dynamics 29:129 (2002).

12. R. Gorenflo, G. D. Fabritiis, and F. Mainardi, Phys. A 269:79 (2004).

13. A. E. Hassan and M. M. Mohamed, J. Hyd. 275:242 (2002).

14. A. Janicki and A. Weron, Simulation and Chaotic Behavior of a-stable Stochastic Processes, p. 355, Marcel Dekker, Inc., New York, 1994.

15. W. Kinzelbach, In: E. Custodio (Ed.), Groundwater Flow and Quality Modeling, pp. 227-245, Reidel Publishing Company, 1988.

16. K. A. Klise, V. C. Tidwell, S. A. McKenna, and M. D. Chapin, Geol. Soc. Am. Abstr. Programs 36(5):573 (2004).

17. E. M. LaBolle, G. E. Fogg, and A. F. B. Tompson, Water Resour. Res. 32:583 (1996).

18. E. M. LaBolle, J. Quastel, G. E. Fogg, and J. Gravner, Water Resour. Res. 36(3):651 (2000).

19. F. Liu, V. Anh, and I. Turner, J. Comput. Appl. Math. 166:209 (2004).

20. M. M. Meerschaert and C. Tadjeran, Appl. Nume. Math. 56:80 (2006).

21. M. M. Meerschaert and C. Tadjeran, J. of Comp. and Appl. Math. 172:65 (2004). 
22. M. M. Meerschaert and H. P. Scheffler, Frac. Cal. Appl. Analy. 5(1):27 (2002).

23. M. M. Meerschaert and H. P. Scheffler, Limit Distributions for Sums of Independent Random Vectors: Heavy Tails in Theory and Practice, pp. 45-46, John Wiley \& Sons, New York, 2001.

24. M. M. Meerschaert, D. A. Benson, and B. Baeumer, Phys. Rev. E 59(5):5026 (1999).

25. M. M. Meerschaert, D. A. Benson, and B. Baeumer, Phys. Rev. E 63(2):12 (2001).

26. M. M. Meerschaert, J. Mortensen, and S. W. Wheatcraft, Phys. A., to appear (2006).

27. R. Metzler and J. Klafter, J. Phys. A. 161:16 (2004).

28. R. Metzler, E. Barkai, and J. Klafter, Europhys. Lett. 46(4):431 (1999).

29. K. S. Miller and B. Ross, An Introduction to Fractional Calculus and Fractional Differential Equations, John Wiley, New York, 1993.

30. T. J. Osler, The Americ. Math. Mon. 78(6):645 (1971).

31. H. Risken, The Fokker-Planck Equation, p. 454, Springer \& Verlag, New York, 1984.

32. J. P. Roop, Ph.D. dissertation, Clemson University, 2004 (unpublished).

33. G. Samorodnitsky and M. Taqqu, Stable Non-Gaussian Random Processes, Chapman \& Hall, New York, 1994.

34. H. Scher and M. Lax, Phys. Rev. B 7(10):4491 (1973).

35. R. Schumer, D. A. Benson, M. M. Meerschaert, and S. W. Wheatcraft, J. Contam. Hyd. 48:69 (2001).

36. I. M. Sokolov and R. Metzler, J. Phys. A 37: L609 (2004).

37. D. Stroock, Wahrscheinlichkeitstheorie verw. Gebiete 32:209 (1975).

38. G. J. M. Uffink, In: H. E. Kobus and W. Kinzelbach (Eds.), Contaminant Transport in Groundwater, p. 283, Brookfield: A.A. Balkema, Vt., 1989.

39. G. S. Weissmann, Y. Zhang, E. M. LaBolle, and G. E. Fogg, Water Resour. Res. 38(10), doi: 10.1029/2001WR000907.

40. V. V. Yanovsky, A. V. Chechkin, D. Schertzer, and A. V. Tur, Phys. A 282:13 (2000). 